\title{
Comparison between Pulsed High-Dose Dexamethasone and Daily Corticosteroid Therapy for Adult Primary Immune Thrombocytopenia: A Retrospective Study
}

\author{
Kumi Nakazaki ${ }^{1,2}$, Masataka Hosoi ${ }^{1}$, Akira Hangaishi ${ }^{1}$, Motoshi Ichikawa ${ }^{1}$, \\ Yasuhito Nannya ${ }^{1}$ and Mineo Kurokawa ${ }^{1}$
}

\begin{abstract}
Objective Recently, pulsed high-dose dexamethasone (HD-Dexa) therapy was proposed as a possible alteration for the classical prednisolone (PSL) therapy for primary immune thrombocytopenia (ITP) patients, however it remains to be confirmed which of these remedies is superior. So the objective of this study is to compare the efficacy and the sustainability of these options.

Methods The first-line therapy at our institute for untreated adult ITP cases was accordingly changed as follows, and we retrospectively evaluated the outcomes: 1) daily administration of $0.5-1 \mathrm{mg} / \mathrm{kg}$ PSL for 2-4 weeks and subsequently stepwise reduction, 2) one course of HD-Dexa (40 mg/day for four consecutive days, 1xHD-Dexa), 3) three courses of the same dose of HD-Dexa (3xHD-Dexa) repeated biweekly. This study was approved by the ethical committee of the University of Tokyo.

Results Twenty-five patients were enrolled consecutively. A good initial response was attained through all the regimens. Meanwhile, time to next treatment for lack of response or relapse was significantly longer in the PSL group than in the other groups (log-rank test, PSL vs. 1xHD-Dexa $\mathrm{p}<0.001$, PSL vs. 3xHD-Dexa $\mathrm{p}=$ 0.0053 , respectively). Additionally, PSL regimen conferred a significantly longer duration time of response (PSL vs. 1 xHD-Dexa $\mathrm{p}=0.0024$, PSL vs. $3 \mathrm{xHD}-\mathrm{Dexa} \mathrm{p}=0.028$, respectively) and CR (PSL vs. 1xHD-Dexa $\mathrm{p}=0.012$, PSL vs. $3 \mathrm{xHD}-\mathrm{Dexa} \mathrm{p}=0.0090$, respectively). No patient discontinued the treatment due to side effects in this study.

Conclusion PSL regimen was considered to be superior to pulsed HD-Dexa regimens in the sustainability of response.
\end{abstract}

Key words: primary immune thrombocytopenia, ITP, high-dose dexamethasone, prednisolone

(Intern Med 51: 859-863, 2012)

(DOI: 10.2169/internalmedicine.51.7005)

\section{Introduction}

Poor long-term sustainability and possible adverse effects by long-time administration of corticosteroids were matters in dispute of the standard corticosteroid therapy for the firstline treatment of adult primary immune thrombocytopenia (ITP) $(1,2)$. Recently, several reports suggested that highdose dexamethasone (HD-Dexa) therapy might be a possible alteration for the classical PSL therapy for newly diagnosed
ITP patients (3-5). A high initial response of a single dose of HD-Dexa therapy was shown by Cheng et al. (4), and the Gruppo Italiano Malattie EMatologiche dell'Adulto (GIMEMA) party demonstrated that three cycles of biweekly HD-Dexa pulses conferred a significantly longer duration of response (6). Indeed, the latest international consensus report and practice guideline for ITP proposed HD-Dexa therapy as one of the front-line options for ITP $(2,6)$.

According to these reports, the front-line therapy at our institute for untreated adult ITP patients was switched from

${ }^{1}$ Department of Hematology and Oncology, Graduate School of Medicine, The University of Tokyo, Japan and ${ }^{2}$ Division for Health Service Promotion, The University of Tokyo, Japan

Received for publication November 25, 2011; Accepted for publication January 9, 2012

Correspondence to Dr. Mineo Kurokawa, kurokawa-tky@umin.ac.jp 
Table 1. Clinical Characteristics of 25 Patients with ITP

\begin{tabular}{|c|c|c|c|c|c|}
\hline \multicolumn{2}{|l|}{$\bar{l}$} & PSL & 1xHD-Dexa & 3xHD-Dexa & $\mathrm{p}$ value \\
\hline \multicolumn{2}{|l|}{ number } & 8 & 12 & 5 & 0.39 \\
\hline \multicolumn{2}{|l|}{ (male/ female) } & $(3 / 5)$ & $(6 / 6)$ & $(4 / 1)$ & \\
\hline \multicolumn{2}{|l|}{ age (years) } & 70.5 & 53.5 & 51 & 0.37 \\
\hline \multicolumn{2}{|l|}{ median (range) } & $(31-86)$ & $(21-75)$ & $(41-61)$ & \\
\hline \multirow{2}{*}{\multicolumn{2}{|c|}{$\begin{array}{l}\text { platelet count }\left(\times 10^{9} / \mathrm{L}\right) \\
\text { median (range) }\end{array}$}} & 5.5 & 7.0 & 5.0 & 0.48 \\
\hline & & $(3-21)$ & $(1-28)$ & $(3-8)$ & \\
\hline \multirow{2}{*}{\multicolumn{2}{|c|}{$\begin{array}{l}\text { H.pyroli } \\
\text { infection* }(\%)\end{array}$}} & 4 & 3 & 2 & 0.56 \\
\hline & & $(50 \%)$ & $(25 \%)$ & $(40 \%)$ & \\
\hline \multirow[t]{5}{*}{ bleeding score ${ }^{\#}$} & 0 & 1 & 2 & 1 & \\
\hline & 1 & 2 & 1 & 1 & \\
\hline & 2 & 5 & 8 & 3 & \\
\hline & 3 & 0 & 1 & 0 & \\
\hline & 4 & 0 & 0 & 0 & \\
\hline
\end{tabular}

PSL indicates prednisolone; 1xHD-Dexa, one course of High-Dose

Dexamethasone; 3xHD-Dexa, three courses of the same dose of

High-Dose Dexamethasone; H.pylori, Helicobacter pylori;

*H. pylori infection was tested by a urea breath test or a blood antibody test.

\# Bleeding score was according to the definition of the report by the GIMEMA group (reference 5).

PSL to HD-Dexa regimens. For lack of a randomized control test comparing the both regimens, we planned this retrospective study to evaluate the outcomes of these alternatives.

\section{Materials and Methods}

\section{Patients}

The newly diagnosed and untreated adult primary ITP patients at our institute from 2000 to 2010 were enrolled consecutively. The diagnosis was based on low platelet count with or without bleeding symptoms and normal to increased number of megakaryocytes in the bone marrow by aspiration. Patients with marrow dysplasia or other causes of thrombocytopenia including auto-immune diseases, liver cirrhosis, and other hematological diseases were excluded.

We applied interventions consecutively for patients with a platelet count of less than $30 \times 10^{9} / \mathrm{L}$ or with bleeding symptoms. We also excluded patients with pregnancy. Patients of secondary thrombocytopenia due to Helicobacter pylori $(H$. pylori) were excluded, who improved by eradication therapy of $H$. pylori despite inadequate recovery by steroid therapy alone, or who maintained platelet response after termination of steroids with successful eradication of $H$. pylori. Infection of $H$. pylori was tested through a urea breath test or a blood antibody test. Bleeding symptoms were classified from grade 0 to 4 according to the definition of the preceding report by the GIMEMA group as follows: 0, Absent bleeding; 1, Petechiae; 2, Ecchymoses and/or dripping with moderate loss of blood; 3, Major mucous hemorrhage with copious loss of blood without sequelae; 4, Major mucous and/or parenchymal hemorrhage with copious loss of blood with sequelae and/or life threatening or death (5).

\section{Study protocol}

The front-line therapies for untreated ITP were altered with time as follows: 1) daily administration of $0.5-1 \mathrm{mg} / \mathrm{kg}$ PSL for 2-4 weeks and subsequent reduction by 5-10 mg per 1-2 weeks to $5-10 \mathrm{mg} /$ day while the platelet count was maintained, then followed by slower reduction of PSL from January 2000 until May 2004, 2) one course of HD-Dexa (40 mg per day for four consecutive days, denoted as 1xHD-Dexa) between June 2004 and March 2009, 3) three courses of the same dose of HD-Dexa (denoted as 3xHDDexa) repeated biweekly after April 2009 until December 2010. In the PSL group, the pace of dose reduction was finally decided at the discretion of attending physicians. Administration of antacids, antibiotics, and other interventions were applied to prevent probable adverse events. This study was approved by the ethical committee of the University of Tokyo.

We primarily evaluated the time to next treatment defined as the interval from the front-line regimens to salvage therapies for lack or loss of response of the former. We also examined response rate and its duration of each regimen according to the recommended criteria (7). Briefly, "response" was defined as any platelet count between 30 and $100 \times 10^{9} /$ $\mathrm{L}$, and at least doubling of the baseline count. "CR" was defined as any platelet count at least $100 \times 10^{9} / \mathrm{L}$. "Response" also required concurrent resolution of bleeding symptoms. Duration time of response or $\mathrm{CR}$ was calculated from the time of response or CR achievement until loss of response.

\section{Statistical analysis}

Fisher's exact test was applied to detect the difference of categorical variables including sex, $H$. pylori infection status, response rate and CR rate among regimens. Analysis of variance was performed for comparing age, platelet count at diagnosis, time span from therapy initiation to response achievement among the three regimens. Time to next treatment and duration time of response and CR were calculated using the Kaplan-Meier method and the difference between the groups was assessed by log-rank test.

\section{Results}

\section{Patients}

Twenty-five patients (13 males and 12 females) were enrolled consecutively in this study, and the median age was 55 years old (range, 21-86). Eight, twelve, and five people received treatment regimens of PSL, 1xHD-Dexa, and 3xHD-Dexa in accordance with the changes of the upfront regimens in the times, respectively (Table 1). However, two patients received PSL due to the complications or age when HD-Dexa was adopted. No significant differences were 

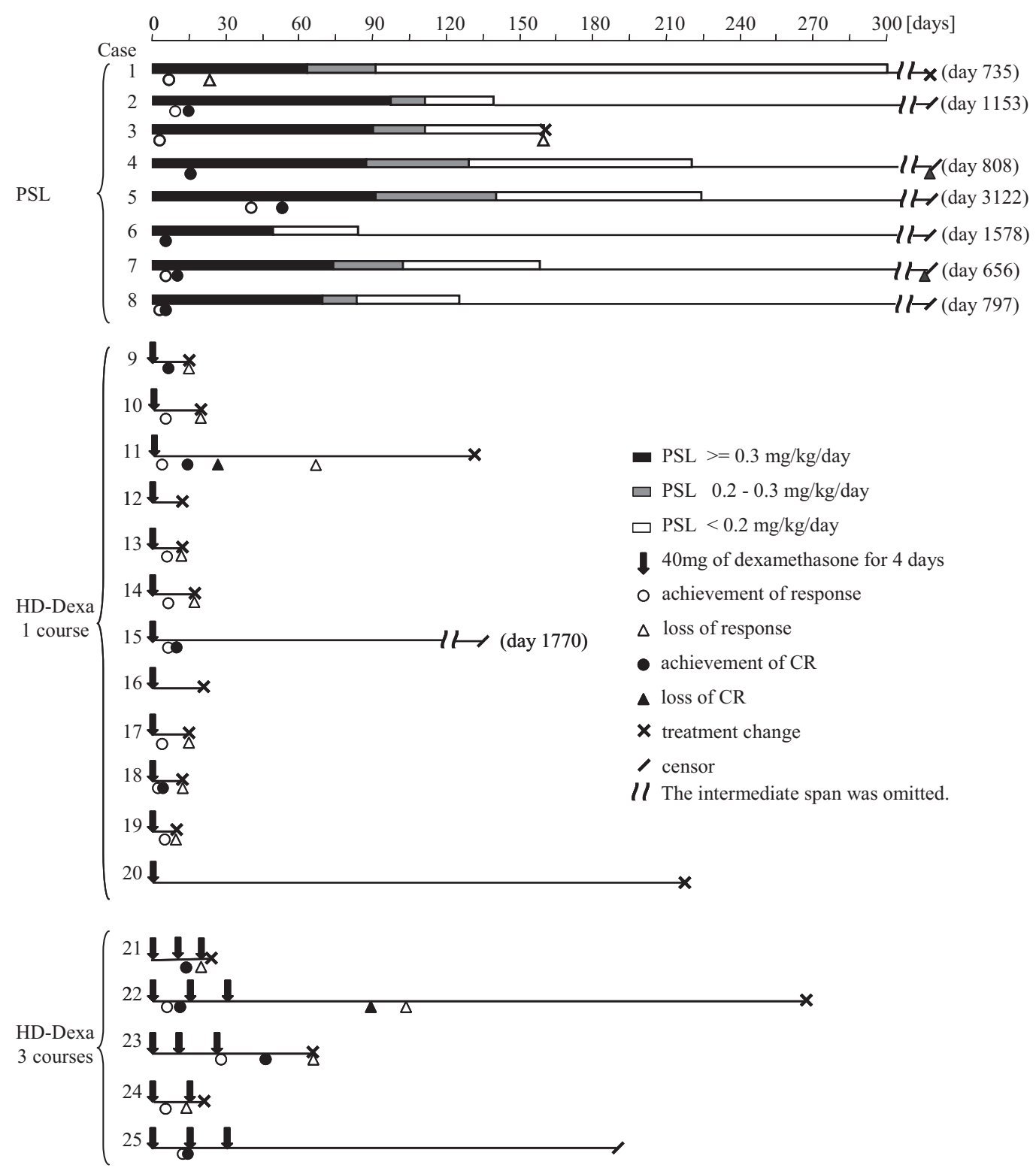

Figure 1. Clinical courses of all twenty-five cases. Eight, twelve and five cases were administered daily prednisolone, one course of $\mathbf{4 0} \mathbf{~ m g}$ of dexamethasone for four consecutive days and three course of the same regimen in times, respectively. Symbols and abbreviations; CR: complete response, HD-Dexa: high-dose dexamethasone, PSL: prednisolone, Each numbered line denotes the clinical course of each case. Each bar of the prednisolone group shows the change of the dose of each case. Black, gray and white colors mean the periods of the dose of $0.3 \mathrm{mg} / \mathrm{kg} / \mathrm{day}$ or more than that, $0.2 \mathrm{mg} / \mathrm{kg} / \mathrm{day}$ or more than that and less than $0.2 \mathrm{mg} / \mathrm{kg} / \mathrm{day}$, respectively. White or black circles display acquirement of response and complete response. White and black triangles mean loss of these responses. A slant bar and a cross indicate the point of censor and change of treatment for each case. Double vertical undulating lines exhibit the case spent without relapse, treatment change or being censored in this span. Each figure in parentheses indicates the day on which the case relapsed or was censored.

found between these three groups in characteristics of patients. Median follow-up time was 32.2 months (range, 1.3102.6 months). The clinical courses of all the cases are shown (Fig. 1). One, one, and two patients underwent eradication of $H$. pylori therapy during their scheduled treatment period in the PSL, 1xHD-Dexa, and 3xHD-Dexa groups, respectively. Eight cases of the PSL group were given median $3,069 \mathrm{mg}(2,225-6,510 \mathrm{mg})$ of PSL in total in the scheduled period. No patients needed concomitant administration of intravenous immunoglobulin in this study.

\section{Efficacy}

A good initial response was achieved through all the regimens. Response rate was PSL: $100 \%$ (95\% confidential interval: $63.1-100 \%)$, 1xHD-Dexa: $75 \%$ (42.8-94.5\%), and 3xHD-Dexa: $100 \%(47.8-100 \%)$, and CR rate was PSL: 
A

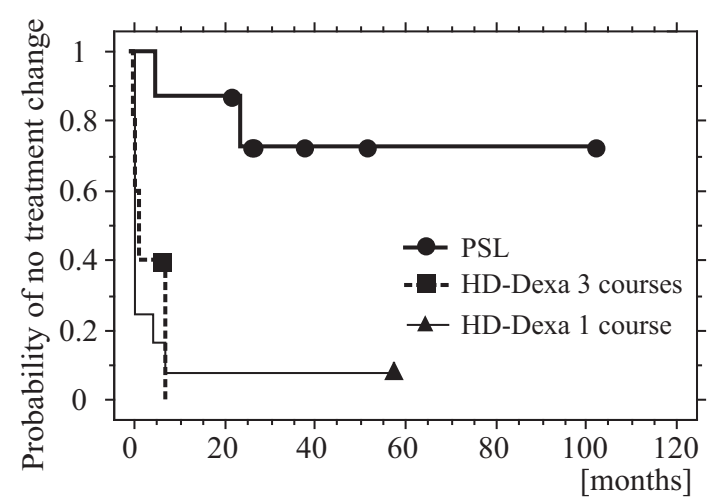

B

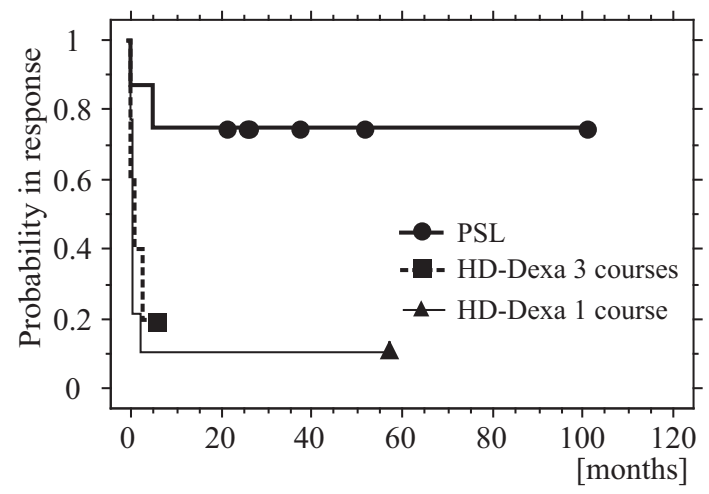

C

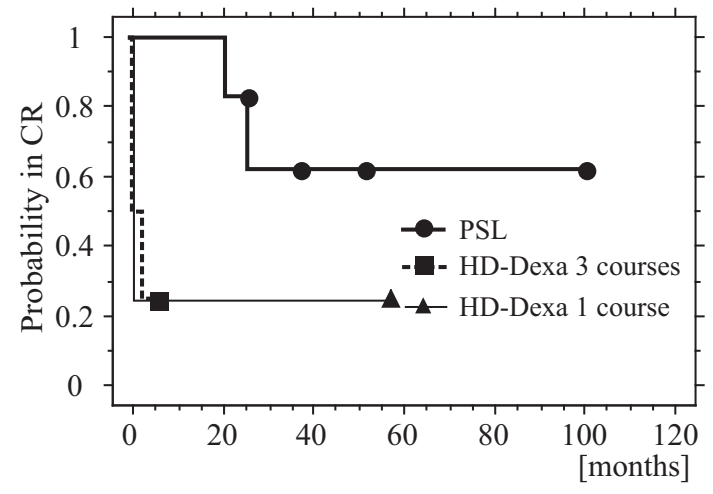

Figure 2. (A) Free from treatment change curves according to the therapy regimens. (B) (C) Duration time of response (B) and complete response $(\mathrm{C})$ according to the therapy regimens. Only responders and complete responders were included in (B) and (C), respectively. PSL indicates prednisolone, HDDexa: high-dose dexamethasone, $\mathrm{CR}$ : complete response.

75\% (34.9-96.8\%), 1xHD-Dexa: $33 \%$ (9.9-65.1\%), and 3 xHD-Dexa: $80 \%$ (28.4-99.5\%), respectively. Meanwhile, CR rate of PSL and 3xHD-Dexa tended to be superior to that of 1xHD-Dexa (PSL vs 1xHD-Dexa $\mathrm{p}=0.17$, 3xHD-Dexa vs 1xHD-Dexa $\mathrm{p}=0.13$ ), and response rate was similar among the three groups. For the responding cases, the initial achievement of response took $11.4 \pm 12.3$ days (average \pm standard deviation), $5.0 \pm 2.0$ days, and $11.8 \pm 8.0$ days since the initiation of the treatment in the PSL, 1xHD-Dexa, and $3 x H D-D e x a$ group, respectively. The span until initial re-
Table 2. Adverse Events

\begin{tabular}{|c|c|c|c|}
\hline & $\begin{array}{l}\text { Number of } \\
\text { (total/ Grad }\end{array}$ & 4) according $t$ & CTCAE ver 4 \\
\hline Adverse events & PSL & 1xHD-Dexa & 3xHD-Dexa \\
\hline Hypertension & $2 / 1$ & $0 / 0$ & $0 / 0$ \\
\hline Hyperglycemia & $2 / 0$ & $1 / 0$ & $1 / 0$ \\
\hline Hyperlipidemia & $1 / 0$ & $0 / 0$ & $0 / 0$ \\
\hline Insomnia & $1 / 0$ & $2 / 0$ & $1 / 0$ \\
\hline Gastrointestinal pain & $0 / 0$ & $1 / 0$ & $0 / 0$ \\
\hline Flushing & $0 / 0$ & $1 / 0$ & $0 / 0$ \\
\hline Others & $1 / 1 *$ & $0 / 0$ & $0 / 0$ \\
\hline \multicolumn{4}{|c|}{ PSL indicates prednisolone; 1xHD-Dexa, one course of High-Dose } \\
\hline \multicolumn{4}{|c|}{ Dexamethasone; 3xHD-Dexa, three courses of the same dose of } \\
\hline \multicolumn{4}{|c|}{ High-Dose Dexamethasone; } \\
\hline \multicolumn{4}{|c|}{ * One patient with cardiac complication before treatment suffered } \\
\hline
\end{tabular}

sponse was not significantly different among the groups ( $\mathrm{p}=$ 0.26 ).

In addition, time to next treatment was significantly longer in the PSL group than in the other groups (PSL vs. $1 \mathrm{xHD}-$ Dexa $\mathrm{p}<0.001$, PSL vs. 3xHD-Dexa $\mathrm{p}=0.0053$, respectively; Fig. 2A). Median of time to next treatment was not reached in the PSL group, and it took 14 days for 1 xHD-Dexa group, and 55 days for 3xHD-Dexa group, respectively. No significant difference was observed between 1xHD-Dexa and 3xHD-Dexa groups $(\mathrm{p}=0.21)$.

Then, sustainability of the effect was assessed. The PSL group exhibited a significantly longer duration time of response than the other two groups (PSL vs. 1xHD-Dexa $\mathrm{p}=$ 0.0024, 3xHD-Dexa vs. 1xHD-Dexa $\mathrm{p}=0.028$ ) (Fig. 2B). Duration time of CR was also significantly longer in this group (PSL vs. $1 \times$ HD-Dexa $\mathrm{p}=0.0090$, PSL vs. $3 \mathrm{xHD}-\mathrm{Dexa}$ $\mathrm{p}=0.012$ ) (Fig. 2C).

Accordingly, a high incidence of early relapse was a cause of treatment change in both of the HD-Dexa groups. In the PSL group, all six cases with achievement of initial CR maintained CR after six months from therapy initiation. Four people with $\mathrm{CR}$ had no need for maintenance therapy at this point, and maintained a response for median 27.7 months (range, 16.4-49.1 months) after termination of therapy. Both of the other two patients in CR state required only $2.5 \mathrm{mg} /$ day dose of PSL at this point. Six out of twelve patients in 1xHD-Dexa group but no patient in the other groups underwent splenectomy through the follow-up period.

\section{Side effects}

In total, fourteen people had some kind of side effect (Table 2). Long-term unfavorable effects were not observed. No patient discontinued the treatment due to side effects related to therapy in this study. 


\section{Discussion}

All three regimens conferred an initial good response, however, time to next treatment of the PSL group was significantly longer than those of the other two groups. This was attributed to a high incidence of early relapse in both of the HD-Dexa groups. Duration time of response and CR was significantly shorter in these groups than the PSL group.

A few studies for untreated ITP patients $(3-5,8,9)$ and for refractory or pre-treated adult ITP patients $(4,10-15)$ have been reported regarding the efficacy of HD-Dexa regimens. Even with the difference of the criteria of response and the number of cycles of HD-Dexa, an initial response was obtained in more than $70 \%$ in the untreated groups. In the present analysis HD-Dexa regimens yielded a similar good response in comparison with historical data $(3-5,8)$. Meanwhile, as to time to next treatment, the duration time was short for the untreated ITP patients. The first-line therapy for untreated ITP patients at our institution was switched back to the PSL regimen considering the advantage of this group in the sustainability of response.

In addition, the rate of initial CR tended to be lower in the one course of HD-Dexa group than in the others. So, both in the initial response rate and sustainability of the obtained response, only one course of the regimen without additional therapy was estimated to be inadequate for treatment of ITP patients. The preceding report showed that repetition of HD-Dexa regimen improved the long-term outcome (5), however, in this study no significant difference was obtained between the HD-Dexa groups in time to next treatment and duration of time of response and CR.

A risk of chronic side effects due to an increased accumulated dose of corticosteroids by long-term administration of the medicine was one of the disputed points in selecting the front-line therapy against ITP. In this study chronic adverse effects did not occur. When the patients of 1xHD-Dexa and 3xHD-Dexa group completed the regimen, $160 \mathrm{mg}$ and 480 $\mathrm{mg}$ of dexamethasone in total were given. These dosages correspond to $1,000 \mathrm{mg}$ and 3,000 $\mathrm{mg}$ of PSL in glucocorticoid activity, respectively. Surprisingly median total dosage given to the PSL group was nearly equal to the converted amount of 3xHD-Dexa group during the scheduled period.

In conclusion, the standard PSL regimen was considered to be superior to HD-Dexa regimens in the sustainability of response, which was compatible with the very recent guideline for ITP (6). The present study suffered retrospective nature and low subject number, thus a prospective randomized study comparing PSL and HD- Dexa regimens is warranted.

The authors state that they have no Conflict of Interest (COI).

\section{Acknowledgement}

We appreciate all the people concerned. K.N., M.H., A.H., and Y.N. designed the protocol and analyzed the data. K.N., M. H., and Y.N. wrote the paper. Y.N., M.I., and M.K. organized the study.

\section{References}

1. Guidry JA, George JN, Vesely SK, Kennison SM, Terrell DR. Corticosteroid side-effects and risk for bleeding in immune thrombocytopenic purpura: Patient and hematologist perspectives. Eur J Haematol 83: 175-182, 2009.

2. Provan D, Stasi R, Newland AC, et al. International consensus report on the investigation and management of primary immune thrombocytopenia. Blood 115: 168-186, 2010.

3. Borst F, Keuning JJ, van Hulsteijn H, Sinnige H, Vreugdenhil G. High-dose dexamethasone as a first- and second-line treatment of idiopathic thrombocytopenic purpura in adults. Ann Hematol 83: 764-768, 2004

4. Cheng Y, Wong RS, Soo YO, et al. Initial treatment of immune thrombocytopenic purpura with high-dose dexamethasone. N Engl J Med 349: 831-836, 2003.

5. Mazzucconi MG, Fazi P, Bernasconi S, et al. Therapy with highdose dexamethasone (HD-DXM) in previously untreated patients affected by idiopathic thrombocytopenic purpura: A GIMEMA experience. Blood 109: 1401-1407, 2007.

6. Neunert C, Lim W, Crowther M, Cohen A, Solberg L Jr, Crowther MA. The American Society of Hematology 2011 evidence-based practice guideline for immune thrombocytopenia. Blood 117: 4190-4207, 2011.

7. Rodeghiero F, Stasi R, Gernsheimer T, et al. Standardization of terminology, definitions and outcome criteria in immune thrombocytopenic purpura of adults and children: Report from an international working group. Blood 113: 2386-2393, 2009.

8. Naithani R, Mahapatra M, Kumar R, Mishra P, Saxena R. High dose dexamethasone therapy shows better responses in acute immune thrombocytopenia than in chronic immune thrombocytopenia. Platelets 21: 270-273, 2010.

9. Pamuk GE, Pamuk ON, Baslar Z, et al. Overview of 321 patients with idiopathic thrombocytopenic purpura. Retrospective analysis of the clinical features and response to therapy. Ann Hematol 81: 436-440, 2002.

10. Stasi R, Brunetti M, Pagano A, Stipa E, Masi M, Amadori S. Pulsed intravenous high-dose dexamethasone in adults with chronic idiopathic thrombocytopenic purpura. Blood Cells Mol Dis 26: 582-586, 2000.

11. Warner M, Wasi P, Couban S, Hayward C, Warkentin T, Kelton JG. Failure of pulse high-dose dexamethasone in chronic idiopathic immune thrombocytopenia. Am J Hematol 54: 267-270, 1997.

12. Arruda VR, Annichino-Bizzacchi JM. High-dose dexamethasone therapy in chronic idiopathic thrombocytopenic purpura. Ann Hematol 73: 175-177, 1996.

13. Caulier MT, Rose C, Roussel MT, Huart C, Bauters F, Fenaux P. Pulsed high-dose dexamethasone in refractory chronic idiopathic thrombocytopenic purpura: A report on 10 cases. Br J Haematol 91: 477-479, 1995.

14. Dubbeld P, van der, Heul C, Hillen HF. Effect of high-dose dexamethasone in prednisone-resistant autoimmune thrombocytopenic purpura (ITP). Neth J Med 39: 6-10, 1991.

15. Andersen JC. Response of resistant idiopathic thrombocytopenic purpura to pulsed high-dose dexamethasone therapy. N Engl J Med 330: 1560-1564, 1994.

(C) 2012 The Japanese Society of Internal Medicine http://www.naika.or.jp/imindex.html 